\title{
Driving impairment due to sleepiness is exacerbated by low alcohol intake
}

\author{
J A Horne, L A Reyner, P R Barrett
}

Occup Environ Med 2003;60:689-692

Aims: To assess whether low blood alcohol concentrations (BACs), at around half the UK legal driving limit, and undetectable by police roadside breathalysers, further impair driving already affected by sleepiness, particularly in young men, who are the most "at risk" group of drivers for having sleep related crashes.

Methods: Twelve healthy young men drove for two hours in the afternoon, in an instrumented car on a simulated motorway. In a repeated measures, counterbalanced design, they were given alcohol or placebo under conditions of normal sleep or prior sleep restriction. Measurements were: driving impairment (lane drifting), subjective sleepiness, and EEG measures of sleepiness.

Results: Whereas sleep restriction and alcohol each caused a significant deterioration in all indices, the combined alcohol and sleep restriction further and significantly worsened lane drifting (which typifies sleep related crashes). This combined effect was also reflected to a significant extent in the EEG, but not with subjective sleepiness. That is, alcohol did not significantly increase subjective sleepiness in combination with sleep loss when compared with sleep loss alone.

Conclusions: Modest, and apparently "safe" levels of alcohol intake exacerbate driving impairment due to sleepiness. The sleepy drivers seemed not to have realised that alcohol had increased their sleepiness to an extent that was clearly reflected by a greater driving impairment and in the EEG.

$\mathrm{D}$ river sleepiness causes about $10 \%$ of all UK road crashes, with a higher rate for monotonous motorways and similar trunk roads. ${ }^{12}$ Such crashes are more likely to result in death or serious injury ${ }^{2}$ owing to a failure to brake beforehand and a higher impact speed. A recent epidemiological study from France ${ }^{3}$ indicated that this outcome is more likely if sleepiness is combined with alcohol, even with blood alcohol concentrations (BACs) as low as $0.010 \mathrm{~g}$ alcohol/100 $\mathrm{ml}$ blood $(0.01 \%)$. The UK alcohol limit for drivers is $0.08 \%$ (equivalent to a breath alcohol concentration of $35 \mu \mathrm{g}$ alcohol/ $100 \mathrm{ml}$ breath). Other EU countries have similar or lower legal limits, and in the USA it is generally $0.10 \%$ for drivers over 21 years, but $0.02 \%$ for those under 21 years.

Three driving studies have combined alcohol with sleepiness. ${ }^{4-6}$ All used: only simple (computer console only) driving simulators, relatively high BACs $(0.05-0.08 \%)$, either minimal $^{6}$ or marked ${ }^{4}$ sleep loss, and only short driving periods. One ${ }^{6}$ was restricted to women (there are sex differences in alcohol elimination ${ }^{7}$ ), and was concerned with circadian effects of alcohol rather than with sleepiness itself. There is the opportunity for a more realistic study involving: a full size car simulator incorporating lengthy and monotonous driving, low BACs well within the "pass" region (BACs $<0.06 \%$ ) of police roadside breathalysers, and a commonly found level of

\section{Main messages}

- Alcohol has a soporific effect which may accentuate any underlying sleepiness in drivers having BACs well within the legal limit. Little research has been directed towards this combination.

- Because of the natural dip in alertness in the afternoon, drivers are more liable to be sleepy at this time, especially after a night of disturbed sleep. Under these conditions, alcohol at lunchtime may be particularly potent.

- In young men (who comprise the group at particular risk of having sleep related road crashes), moderate sleep loss combined with BACs well within the "pass" region for police roadside breathalysers, produces a marked worsening of driving impairment compared with either sleep loss or alcohol alone. While the EEG also reflected this worsened combined effect, this was not so apparent subjectively.

- When combined with sleepiness, apparently "safe" amounts of alcohol impair driving to a greater extent than is reflected in changes to one's perception of the sleepiness.

\section{Policy implications}

- Even a small ("legally passable") intake of alcohol is dangerous for drivers if they are sleepy.

- Sleepy drivers should avoid all alcohol lof course, sleepy drivers shouldn't drive, anyway).

sleepiness, typified in the afternoon and following a night of curtailed sleep. As well as having these features, our study focused on young men, as most (90\%) sleep related crashes in the UK are caused by men, half of whom are under 30 years. ${ }^{2}$

The afternoon is a time of the day when there is a natural circadian dip in alertness, and when sleepiness related crashes tend to increase. ${ }^{2}$ It also follows after a young male driver mindful of the drink driving limits may have had only a modest amount of alcohol during a light lunch.

\section{METHODS}

\section{Participants}

Twelve 12 men (mean age 22.7 years, range 20-26) were recruited by advert and screened by interview. They were healthy and medication-free, with a mean weight of $74.8 \mathrm{~kg}$ (range 69-89 kg), and a mean body mass index (BMI) of 23.0

Abbreviations: BAC, blood alcohol concentration; BMI, body mass index; EEG, electroencephalogram; EOG, electro-oculogram 
(range 20-27). All were experienced drivers (having driven for over two years, for more than three hours per week). They were good sleepers (no sleep complaints), with a mean nighttime sleep length of 503 minutes (range 450-540 minutes), slept regular hours, scored below 10 on the Epworth Sleepiness Scale, ${ }^{8}$ took infrequent daytime naps (less than once per month), and were moderate (2-4 cups daily) drinkers of caffeinated coffee. They had the procedures fully explained, signed consent forms, and were paid to participate. The study was approved by the university ethical committee.

\section{Design and procedure}

There were four conditions, in a within-subjects, double blind (for alcohol), counterbalanced design, with each individual undergoing a different treatment order:

- Normal night sleep + lunchtime alcohol (ALC)

- Normal night sleep + nil alcohol control (BASE)

- Night sleep reduced to five hours + alcohol (SR+ALC)

- Night sleep reduced to five hours + nil alcohol (SR).

The four conditions were given at weekly intervals, following an earlier, 30 minute practice drive. There was nil alcohol intake for 36 hours before each study and nil caffeinated drinks after 1800 the evening before. Participants arrived at the laboratory at 1300 and provided a urine sample as a check (6 Drug Multi Test 1, Surescreen Diagnostics, Derby) for recent recreational drug use. Alcohol comprised $300 \mathrm{ml}$ orange juice with $75 \mathrm{ml}$ of $37.5 \%$ proof vodka, consumed at 1315-1330 after participants had eaten two standard cheese rolls. Food intake slows alcohol absorption, thus reducing BAC. ${ }^{9}{ }^{10}$ Drivers were breathalysed using a calibrated alcometer (model SD400, Lion Laboratories, Glamorgan) at 1355 and again at 1600, following a two hours continuous drive (see below), commencing at 1400. A pilot study breathalysing four similar participants every five minutes between these two times, showed that BACs subsequently did not rise above the 1355 level. Alcohol was not given in proportion to body weight, but as a set volume ( $75 \mathrm{ml}$; approximately three units), as this is more typical of alcohol consumption. It should be noted that when alcohol is given in proportion to body weight, there is little consistency in BACs, and neither is there much consistency for a particular BAC to produce a reliable amount of performance impairment between people. ${ }^{11}$ Nevertheless, to reduce individual differences in blood dilution effects with BACs etc, participants were recruited within a limited body weight/BMI range.

Wrist actimeters are good indicators of sleep and wakefulness, ${ }^{12}$ and participants wore these on the night before each condition, to enable us to monitor compliance with night-time sleep requirements (normal sleep time) or, in the case of sleep restriction, that "lights out" was delayed to ensure five hours sleep with a normal rising time (for example, 0200-0700). Actimeters were checked for participant compliance before each driving session commenced.

\section{Simulator}

This comprised an immobile car with an interactive full size computer generated dull, monotonous roadway having some gradual bends, projected on to a $2.0 \mathrm{~m} \times 1.5 \mathrm{~m}$ screen located $2.3 \mathrm{~m}$ from the windscreen. ${ }^{13}$ There were two "up" and two "down" lanes, hard shoulder, and simulated auditory "rumble strips". Participants sat in the driving seat and drove at their normal cruising speed within white lane markings. Lane drifting is the usual manifestation of sleepy driving. A car wheel crossing a lateral lane marking was identified as a driving "incident". An unobtrusive infrared camera filmed the driver's face, which was recorded with the roadway using a split screen video display. These video data were analysed by a skilled assistant "blind" to the experimental conditions to see whether identified incidents were due: (1) to poor steering (for example, driver taking one hand off steering wheel) or driver distraction (looking elsewhere), both of which were discounted; or (2) to episodes associated with sleepiness (that is, eye closure or vacant staring ahead), which were logged. As a further guide, the electroencephalogram (EEG) and electrooculogram (EOG) (see below) were checked, as: typically during ( 1 ) there is little alpha and theta EEG activity, and with saccadic eye movements; whereas in (2) either or both these EEG activities are present, often with slow "eye rolling" on the EOG. Additional quality checks on these video, EEG and EOG data were undertaken "blind" by a second investigator. Although driver distraction, as in (1) could also be associated with sleepiness, we only logged data that clearly indicated sleepiness, as in (2). Besides, distractions apparently associated with sleepiness would usually be followed by clearer, identifiable signs of sleepiness.

\section{Subjective sleepiness}

Every 200 seconds, participants were asked to respond verbally with a number from the nine point Karolinska Sleepiness Scale ${ }^{14}: 1=$ extremely alert, $2=$ very alert, $3=$ alert, $4=$ rather alert, $5=$ neither alert nor sleepy, $6=$ some signs of sleepiness, 7 = sleepy, no effort to stay awake, $8=$ sleepy, some effort to stay awake, $9=$ very sleepy, great effort to keep awake, fighting sleep. The scale and descriptors were printed on the car's dashboard, within easy view of the driver.

\section{EEG and EOG}

Electrodes were attached for one channel of EEG $\left(\mathrm{C}_{3}-\mathrm{A}_{1}\right)$. To identify "eye rolling" further, two channels of EOG were recorded (electrodes $1 \mathrm{~cm}$ lateral to and $\mathrm{l} \mathrm{cm}$ above left outer canthus, and $1 \mathrm{~cm}$ lateral to and $1 \mathrm{~cm}$ below right outer canthus; both referred to the centre of the forehead). The digitised EEG, sampled at $128 \mathrm{~Hz}$, was recorded using Labview (National Instruments Inc., New Jersey) and spectrally analysed using Somlologica (Embla, Flaga Medical Devices, Iceland) in four-second periods. Low and high band-pass filtering of the EEG at $>30 \mathrm{~Hz}$ and $<0.3 \mathrm{~Hz}$ removed slow eye movements and muscle artifact. ${ }^{13}$ There was some unavoidable eye blink contamination on the EEG, which was mostly filtered out, and does not bias the EEG outcomes. ${ }^{13}$ Increases in EEG power in the alpha $(8-11 \mathrm{~Hz})$ and theta $(4-7 \mathrm{~Hz})$ ranges indicate increasing sleepiness. ${ }^{14}{ }^{15}$ EEG power in this (4-11 Hz) frequency range was then averaged in one-minute epochs. To remove individual differences in these EEG power levels and to permit better comparison between conditions, these data were standardised for each participant by taking the difference between each epoch and an initial mean value for that person's EEG power, divided by the standard deviation around that mean. ${ }^{13}$

\section{Statistical analysis}

Driving incidents, EEG, and subjective sleepiness data were averaged into 30 minute periods per participant and condition, and two-way (condition $\times$ time) repeated measures ANOVAs were applied (using the Huynh-Feldt $(\epsilon)$ adjustment). We used this ANOVA design as we wished to make direct comparisons between all conditions. Tukey post hoc tests were applied where appropriate, and findings better than $\mathrm{p}<0.05$ are reported.

\section{RESULTS}

BACs before and after the drive respectively, showed no significant differences between the two alcohol conditions (table 1). For sleep related driving incidents (fig 1) there was a significant between-conditions effect $(F=11.3$, df 3,33; $\mathrm{p}<0.001, \epsilon=1.0$ ), with post hoc tests showing that all three experimental conditions were significantly greater than BASE, and that SR+ALC was significantly greater than both SR and ALC alone. There was also a significant effect of time 
Table 1 BACs (mg alcohol/100 ml blood) estimated from breath alcohol concentrations

\begin{tabular}{lll}
\hline & Before drive & After drive \\
\hline With sleep restriction & $38.3(4.0)$ & 0 \\
No sleep restriction & $32.4(4.0)$ & 0 \\
\hline
\end{tabular}

Results expressed as mean (SE).

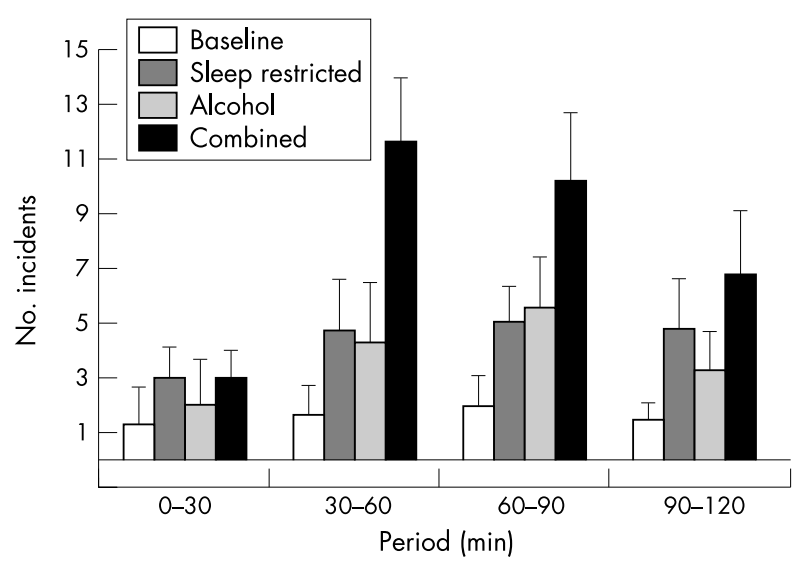

Figure 1 Mean (SE) of sleep related driving incidents (lane drifting) over the four consecutive 30 minute periods, for the four conditions.

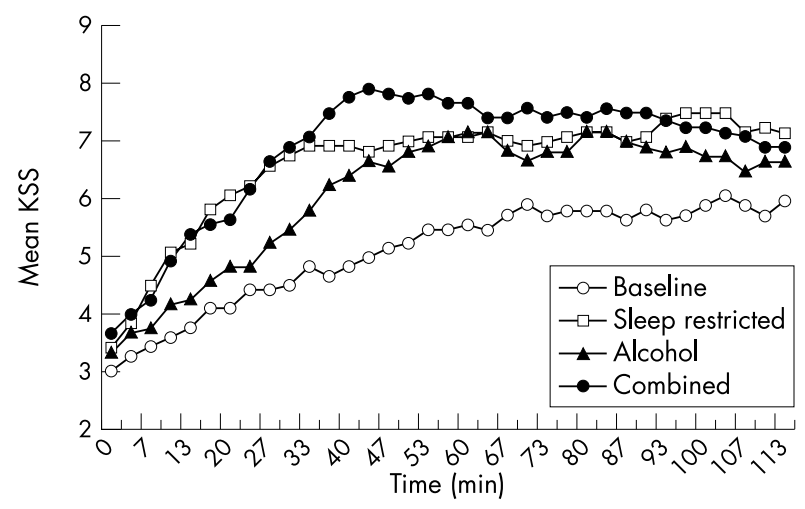

Figure 2 Mean subjective sleepiness ratings over the two hour drive, per 200 seconds, for each condition. KSS, Karolinska Sleepiness Scale.

$(\mathrm{F}=4.6$, df 3,33; $\mathrm{p}<0.01, \epsilon=1.0)$, with post hoc tests revealing the $0-30$ minute period to be significantly lower than all other periods. The conditions $\times$ time interaction was significant $(\mathrm{F}=2.4$, df 9,99; $\mathrm{p}<0.04, \epsilon=0.6)$. Post hoc tests were significant for: the 30-60 min period, when SR + ALC was greater than the other conditions; and for the 60-90 min period, when SR+ALC was greater than BASE.

Subjective sleepiness (fig 2 ) showed a significant betweenconditions effect ( $\mathrm{F}=10.0$, df 3,33; $\mathrm{p}<0.001, \epsilon=1.0)$. Post hoc tests showed that all three experimental conditions were greater than BASE, and that SR and SR+ALC were greater than ALC. Interestingly, however, there was no significant difference between SR and SR+ALC. Time was significant $(\mathrm{F}=19.4$, df 3,33; $\mathrm{p}<0.001, \epsilon=0.44)$, with post hoc tests significant for the 0-30 minute period being lower than the other three. There was no significant interaction between conditions and time.

The EEG from two participants was faulty and not able to be analysed, leaving $\mathrm{n}=10$ for this data set. The outcome from the EEG (fig 3 ) was similar to the other findings. There was a significant between-conditions effect $(\mathrm{F}=4.8$, df 3,27;

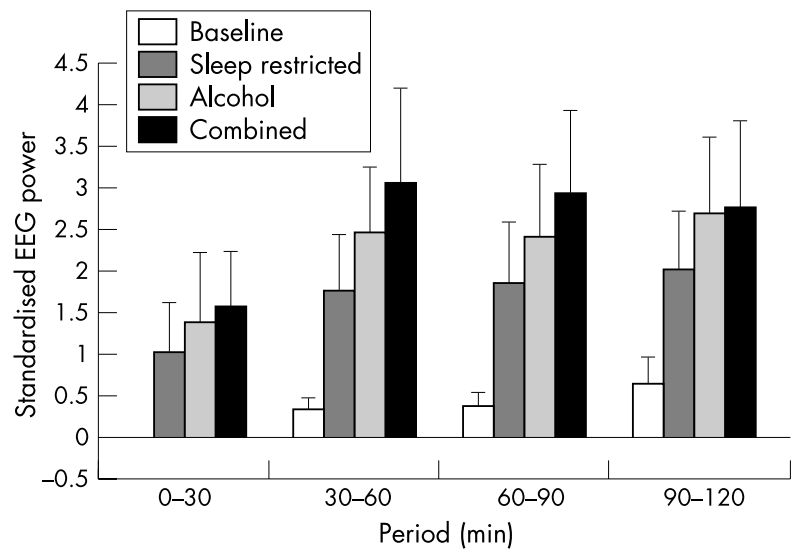

Figure 3 Mean (SE) of alpha + theta EEG power (indicative of sleepiness) $(n=10)$ over the four consecutive 30 minute periods, for the four conditions.

$\mathrm{p}<0.017, \epsilon=0.74)$. Post hoc tests were significant for all three experimental conditions being greater than BASE, and $\mathrm{SR}+\mathrm{ALC}$ greater than SR. Time was significant $(\mathrm{F}=11.7, \mathrm{df}$ 3,27; $\mathrm{p}<0.003, \epsilon=0.44$ ), with post hoc tests showing the 0-30 minute period to be lower than the rest. There was no significant interaction between condition and time.

\section{DISCUSSION}

During the afternoon circadian "dip", and following some sleep restriction the previous night, all three indices of sleepiness (lane drifting, subjective sleepiness, and the EEG) were significantly impaired in our young male drivers. These decrements were similar to those found following normal sleep plus a moderate alcohol intake, giving BACs well within the "pass" limit for UK police roadside breathalysers. However, when the sleep restriction and alcohol were combined, there was a marked, further worsening of lane drifting. On the other hand, for subjective sleepiness this effect of the combination was not so pronounced, even though the EEG also revealed a significant change from the sleep restriction only condition.

Given that the level of subjective sleepiness produced by the combined effect was somewhat similar to that for the sleep restriction alone, whereas for lane drifting the combined effect was far more evident, then sleepy drivers having consumed some alcohol may not realise how bad their driving is, if they rely on sleepiness as a guide to driving impairment. However, it must be remembered that we only studied young men, as they are the most at risk group for sleep related crashes. These drivers generally tend to perceive most adverse driving situations to be less risky and accidents less likely. ${ }^{16}$

In conclusion, because of the natural afternoon "dip" in alertness, even after a normal night's sleep, a modest alcohol intake at lunchtime (giving BACs well within the "pass" range for police roadside breathalysers) presents a potential danger to driving at this time, especially under dull and monotonous conditions. This hazard with alcohol is markedly worsened if the afternoon sleepiness was further enhanced by sleep disturbance the previous night.

With the increasing pressures in today's society causing people to reduce the time they spend asleep at night, they are more likely to experience higher levels of afternoon sleepiness, and place themselves at a greater risk of having a sleep related crash if they also choose to drink any alcohol beforehand.

\section{ACKNOWLEDGEMENTS}

This research was funded by the UK Department of Transport. We would like to thank Stuart Baulk and Adrian Bailey for their help. 


\section{Authors' affiliations}

J A Horne, L A Reyner, P R Barrett, Sleep Research Centre, Loughborough University

Correspondence to: Professor J A Horne, Sleep Research Centre Loughborough University, Loughborough, Leicestershire LE 11 3TU, UK; j.a.horne@lboro.ac.uk

Accepted 12 July 2002

\section{REFERENCES}

1 DTLR. Sleep-related vehicle accidents on sections of trunk roads and motorways in the UK (1995-1998). Road Safety Research Report No. 22. DLTR, 2001.

2 Horne JA, Reyner LA. Sleep related vehicle accidents. BM 1995;310:556-67.

3 Philip P, Vervialle F, Le Breton P, et al. Fatigue, alcohol and serious road crashes in France: factorial study of national data. BM 2001;322:829.

4 Roehrs T, Beare D, Zorick F, et al. Sleepiness and ethanol effects on simulated driving. Alcoholism Clin Exper Res 1994;18:154-8.

5 Arnedt JT, Wilde GJS, Munt PW, et al. Simulated driving performance following prolonged wakefulness and alcohol consumption: separate and combined contributions to impairment. J Sleep Res 2000;9:233-41.

6 Horne JA, Baumber CJ. Time-of-day effects of alcohol intake on simulated driving performance in women. Ergonomics 1991;34:1377-83.
7 Thomasson $\mathbf{H}$. Alcohol elimination: faster in women? Alcoholism Clin Exper Res 2000;24:419-20.

8 Johns MW. A new method for measuring daytime sleepiness: the Epworth Sleepiness Scale. Sleep 1991;14:540-6.

9 Millar K, Hammersley RH, Finnigan F. Reduction of alcohol-induced performance impairment by prior ingestion of food. Br J Psychol 1992;83:261-78.

10 Horne JA, Gibbons $\mathrm{H}$. Effects on vigilance performance and sleepiness of alcohol given in the early afternoon ("post lunch") vs. early evening. Ergonomics 1991;34:67-77.

11 Moskowitz H, Robinson C. Driving-related skills impairment at low blood alcohol levels. In: Noordzij PC, Roszbach R, eds. Alcohol, Drugs and Traffic Safety-T86. Amsterdam: Excerpta Medica, 1986:79-86.

12 Horne JA, Pankhurst FL, Reyner LA, et al. A field study of sleep disturbance: effects of aircraft noise and other factors on 5,742 nights of actimetrically monitored sleep in a large subject sample. Sleep 1994; 17:146-59.

13 Horne JA, Reyner LA. Counteracting driver sleepiness: effects of napping, caffeine and placebo. Psychophysiology 1996;33:306-9.

14 Åkerstedt T. Gillberg M. Subjective and objective sleepiness in the active individual. Int J Neurosci 1990;52:29-37.

15 Rechtschaffen A, Kales A. A manual of standardised terminology techniques, and scoring system for sleep stages of human subjects. Los Angeles: UCLA Brain Information Service, 1968.

16 Leigh BC. Peril, chance, adventure: concepts of risk, alcohol use and risky behaviour in young adults. Addiction 1999;94:371-83.

We are delighted to announce this forthcoming conference in Auckland, New Zealand.

The themes of the 3rd Asia Pacific Forum on Quality Improvement in Health Care are:

- Agenda for quality: Improving equity in health care delivery

- Improving safety

- Leadership for improvement

- Measuring quality and benchmarking for change

- Evidence based knowledge and education for quality improvement

- Improving health systems

- Patient/consumer centred quality improvement

Presented to you by the BM Publishing Group (London, UK) and Institute for Healthcare Improvement (Boston, USA), supported by the New Zealand Ministry of Health, ACC, and Standards New Zealand.

For more information about the Forum or to register contact: quality@bma.org.uk or go to: www.quality.bmipg.com

Tel: +44 (0)2073836409 Fax: +44 (0)2073836869 